\title{
Dexamethasone Implant as Sole Therapy in Sympathetic Ophthalmia
}

\author{
Ahmad M. Mansour \\ Department of Ophthalmology, American University of Beirut, Beirut, Lebanon; \\ Department of Ophthalmology, Rafic Hariri University Hospital, Beirut, Lebanon
}

\section{Keywords}

Dexamethasone implant · Miosis · Sympathetic ophthalmia $\cdot$ Uveitis

\begin{abstract}
We present the case of a 46-year-old woman with sympathetic ophthalmia occurring 27 years after complicated juvenile cataract surgeries. The patient declined systemic immunosuppressive therapy. Dexamethasone implant in the sympathizing eye allowed good visual recovery up to 18 months of follow-up with a total of 6 implants. Intraocular pressure rise was controlled medically. This is a unique report of sympathetic ophthalmia treated solely with slow-release dexamethasone implant without systemic therapies.

(C) 2018 The Author(s)

Published by S. Karger AG, Basel
\end{abstract}

\section{Introduction}

Sympathetic ophthalmia (SO) is traditionally managed with systemic immunosuppression [1-11]. We report our experience using intravitreal dexamethasone implant in the treatment of sympathetic uveitis. 


\section{Case Reports in Ophthalmology}

\section{Case Report}

This 46-year-old Caucasian woman presented with painful visual loss in the left eye of 1 week. The left eye was rendered aphakic after surgery for autosomal dominant cataract at the age of 15 years. Simultaneously, the management of the right-eye cataract was much complicated (primary lens aspiration, secondary lens implantation, vitrectomy) by intraocular lens dislocation and retinal detachment. One year before the current presentation (26 years after surgical interventions), she was advised to have enucleation of the painful blind right eye. Corrected visual acuity of the left eye was 20/70 with mutton-fat keratic precipitates, 2+ anterior and posterior segment cells and flare, along with serous retinal detachment of the papillomacular region (Fig. 1). Intravenous fluorescein angiography showed multiple hyperfluorescent leakage sites at the level of the retinal pigment epithelium with subretinal pooling of dye in the papillomacular region (Fig. 2). She underwent enucleation of the right nonseeing eye. There was diffuse lymphocytic infiltration of the choroid in the enucleated right eye. The rheumatologist ordered initiation of immunosuppressive therapy. The patient refused receiving immunosuppressive therapy categorically and repeatedly at every visit. She signed a formal consent for receiving intravitreal dexamethasone implant (Ozurdex; Allergan Inc., Irvine, CA, USA) with knowledge of its various complications and of the contraindication of its use in aphakia. The intravitreal injection procedure was carried out under topical anesthesia and povidone-iodine $5 \%$ irrigation. The sclera was engaged at an oblique angle for approximately $1 \mathrm{~mm}$ and advanced until the vitreous cavity was entered. Visual recovery to $6 / 7.5(20 / 25)$ was achieved, and the implant was injected every 3 months. The left eye was monitored monthly and weekly near to the third month after injection to determine the timing of implant injection. Reinjection was done at the first sign of inflammation using funduscopy (Fig. 3), slit lamp biomicroscopy (detection of flare and cells), anterior optical coherence tomography (OCT) for new onset of keratic precipitates (Fig. 4), and macular OCT (Fig. 1) to detect foveal thickening. We followed the position of the implant by anterior OCT (Fig. 4) and prevented anterior migration by topical pilocarpine $2 \%$ and avoidance of bending forward. Intraocular pressure rose to $27 \mathrm{~mm} \mathrm{Hg}$ after the fourth injection and was controlled medically. The patient repeatedly refused immunosuppression despite the risk of endophthalmitis, glaucoma, and anterior implant migration. Systemic uveitis workup was negative (including chest radiograph, purified protein derivative skin test, venereal disease research laboratory, rheumatoid factor, and antinuclear antibody). She received a total of 6 implants over 18 months of followup, achieving a final visual acuity of 20/25 and an intraocular pressure of $20 \mathrm{~mm} \mathrm{Hg}$.

\section{Discussion}

The dexamethasone intravitreal implant is a biodegradable polymer composed of $0.7 \mathrm{mg}$ of dexamethasone and a polylactic acid-co-glycolic acid. As the implant gradually disintegrates, dexamethasone is slowly released within the vitreous over a 3- to 4-month period [12]. The device has been approved by the US Food and Drug Administration for use in the treatment of macular edema due to diabetic maculopathy [13] or retinal vein occlusions [14] and for noninfectious posterior uveitis in adults [15]. Complications of the implant have included implant migration into the anterior chamber in aphakic eyes, increased intraocular pressure, and cataract progression. Intraocular pressure was well controlled medically in our case. Anterior migration of the implant was prevented by pharmacologic miosis and proper positioning of the face (avoid face-down position). 
SO is a rare nonnecrotizing diffuse granulomatous uveitis following ocular trauma. It appears that disrupted integrity of the inciting traumatized eye leads to an autoimmune hypersensitivity reaction or cell-mediated immune response directed against retina self-antigens in both eyes. Recently, vitreoretinal surgery [10] had overtaken nonsurgical trauma as the major cause of SO [11]. A good visual outcome is achieved with early diagnosis and prompt aggressive immunosuppressive treatment $[2,3]$. Enucleation of the inciting eye is much recommended if done in the first 10 days after the inciting event and when there is no visual potential [6]. The treatment of SO is primarily medical. The mainstay of treatment is systemic immunomodulatory therapy. Systemic corticosteroids are the first-line therapy for SO [1]. If patients are nonresponsive to steroid therapy or have clinically significant side effects, cyclosporine, azathioprine, or other immunosuppressive agents can be used for long-term immunomodulatory therapy. The minimum dosing schedule required to control inflammation is at least 1 year in most cases. Shorter courses have been reported [4,5]. According to Mahajan et al. [9], fluocinolone acetonide implant provides inflammatory control and reduces the dependence on systemic immunosuppression in 8 patients with SO followed between 6 months and 2 years. All patients had significant reduction in the systemic medication used to control inflammation. Two patients had recurrent inflammatory episodes requiring the resumption of an oral immunosuppressive. Vision improved or was stabilized in all 8 patients. The second report by Almeida et al. [8] was of a positive anti-inflammatory response to fluocinolone implant that migrated anteriorly causing corneal edema and necessitating removal of the implant. In this case, uveitis was well controlled by 3 sequential fluocinolone acetonide implant surgeries over 8 years; the implants were separated by 5 years and 3 years with the late dislocation occurring 4 years after original implantation. Our case used dexamethasone implant as the primary therapy without concomitant immunosuppressive therapy and with good control. Cure was not reached with the implant as there was recurrence of inflammation beyond the therapeutic period of 3 months. Longer follow-up of several years using the dexamethasone implant can shed more light on the role of the implant in SO.

\section{Statement of Ethics}

This study followed the tenets of the Declaration of Helsinki. Personal identifiers were removed because consent to publishing such information was not obtained. The study was approved by the Institutional Review Board at Rafic Hariri University Hospital.

\section{Disclosure Statement}

The author has no conflicts of interest to disclose. This is an unfunded study.

\section{References}

1 Arevalo JF, Garcia RA, Al-Dhibi HA, Sanchez JG, Suarez-Tata L. Update on sympathetic ophthalmia. Middle East Afr J Ophthalmol. 2012 Jan;19(1):13-21.

2 Payal AR, Foster CS. Long-term drug-free remission and visual outcomes in sympathetic ophthalmia. Ocul Immunol Inflamm. 2017 Apr;25(2):190-5.

3 Patel SS, Dodds EM, Echandi LV, Couto CA, Schlaen A, Tessler HH, Goldstein DA. Long-term, drug-free remission of sympathetic ophthalmia with high-dose, short-term chlorambucil therapy. Ophthalmology. 2014 Feb;121(2):596-602. 
4 Tessler HH, Jennings T. High-dose short-term chlorambucil for intractable sympathetic ophthalmia and Behçet's disease. Br J Ophthalmol. 1990 Jun;74(6):353-7.

5 Yang CS, Liu JH. Chlorambucil therapy in sympathetic ophthalmia. Am J Ophthalmol. 1995 Apr;119(4):482-8.

6 Reynard M, Riffenburgh RS, Maes EF. Effect of corticosteroid treatment and enucleation on the visual prognosis of sympathetic ophthalmia. Am J Ophthalmol. 1983 Sep;96(3):290-4.

7 Chang GC, Young LH. Sympathetic ophthalmia. Semin Ophthalmol. 2011 Jul-Sep;26(4-5):316-20.

8 Almeida DR, Chin EK, Mears K, Russell SR, Mahajan VB. Spontaneous dislocation of a fluocinolone acetonide implant (Retisert) into the anterior chamber and its successful extraction in sympathetic ophthalmia. Retin Cases Brief Rep. 2015;9(2):142-4.

9 Mahajan VB, Gehrs KM, Goldstein DA, Fischer DH, Lopez JS, Folk JC. Management of sympathetic ophthalmia with the fluocinolone acetonide implant. Ophthalmology. 2009 Mar;116(3):552-557.e1.

10 Pollack AL, McDonald HR, Ai E, Green WR, Halpern LS, Jampol LM et al. Sympathetic ophthalmia associated with pars plana vitrectomy without antecedent penetrating trauma. Retina. 2001;21(2):146-54.

11 Su DH, Chee SP. Sympathetic ophthalmia in Singapore: new trends in an old disease. Graefes Arch Clin Exp Ophthalmol. 2006 Feb;244(2):243-7.

12 Chang-Lin JE, Attar M, Acheampong AA, Robinson MR, Whitcup SM, Kuppermann BD et al. Pharmacokinetics and pharmacodynamics of a sustained-release dexamethasone intravitreal implant. Invest Ophthalmol Vis Sci. 2011 Jan;52(1):80-6.

13 Boyer DS, Yoon YH, Belfort R Jr, Bandello F, Maturi RK, Augustin AJ et al.; Ozurdex MEAD Study Group. Three-year, randomized, sham-controlled trial of dexamethasone intravitreal implant in patients with diabetic macular edema. Ophthalmology. 2014 Oct;121(10):1904-14.

14 Haller JA, Bandello F, Belfort R Jr, Blumenkranz MS, Gillies M, Heier J et al.; OZURDEX GENEVA Study Group. Randomized, sham-controlled trial of dexamethasone intravitreal implant in patients with macular edema due to retinal vein occlusion. Ophthalmology. 2010 Jun;117(6):1134-1146.e3.

15 Lowder C, Belfort R Jr, Lightman S, Foster CS, Robinson MR, Schiffman RM et al.; Ozurdex HURON Study Group. Dexamethasone intravitreal implant for noninfectious intermediate or posterior uveitis. Arch Ophthalmol. 2011 May;129(5):545-53. 


\section{Case Reports in Ophthalmology}
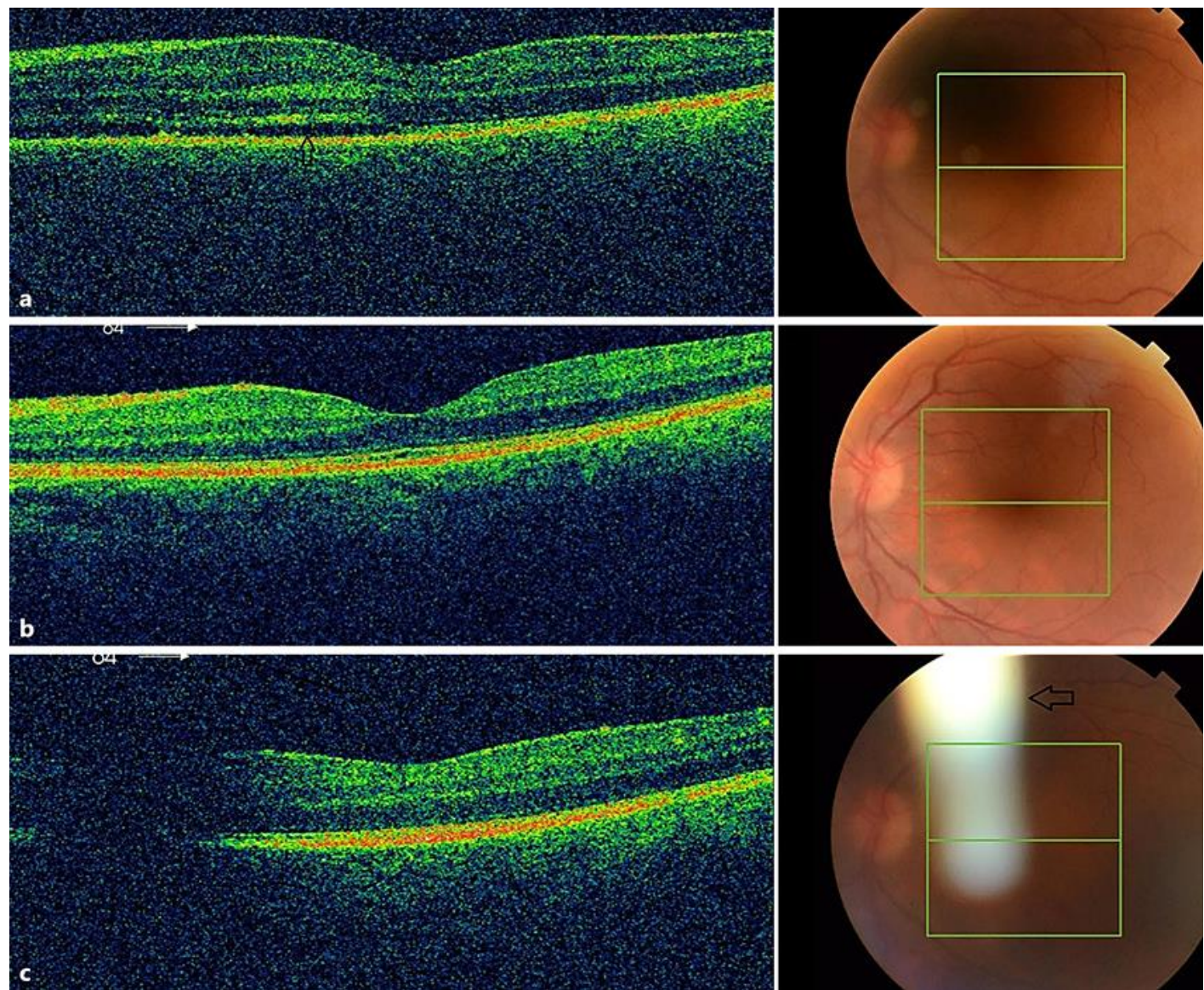

Fig. 1. On presentation, macular spectral-domain OCT (Topcon 3D OCT-2000 FA plus, Topcon Inc., Tokyo, Japan) shows serous elevation (vertical arrow) of the sensory retina (a) that resolved after intravitreal dexamethasone injection (b) with the implant (horizontal arrow) occasionally blocking part of the central field and altering the OCT exam (c). OCT, optical coherence tomography. 


\section{Case Reports in Ophthalmology}

Mansour: Dexamethasone Implant as Sole Therapy in Sympathetic Ophthalmia

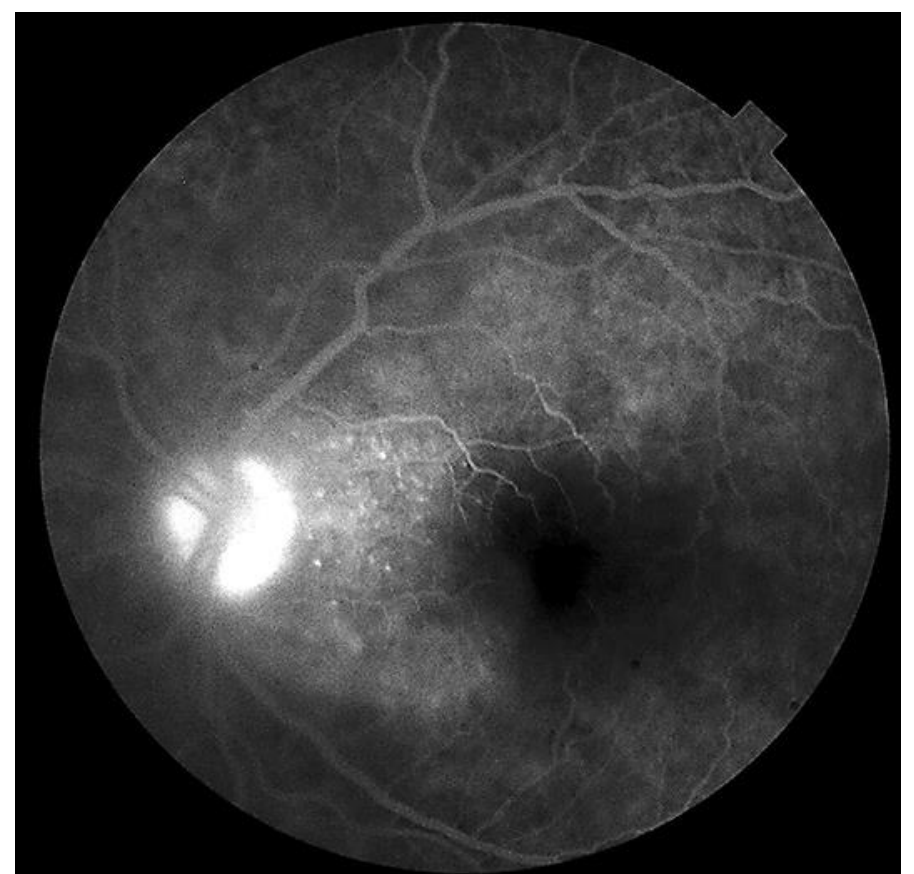

Fig. 2. Venous phase of the fluorescein angiogram of the left eye. There are multiple sites of subretinal dye leakage most marked in the temporal peripapillary area.
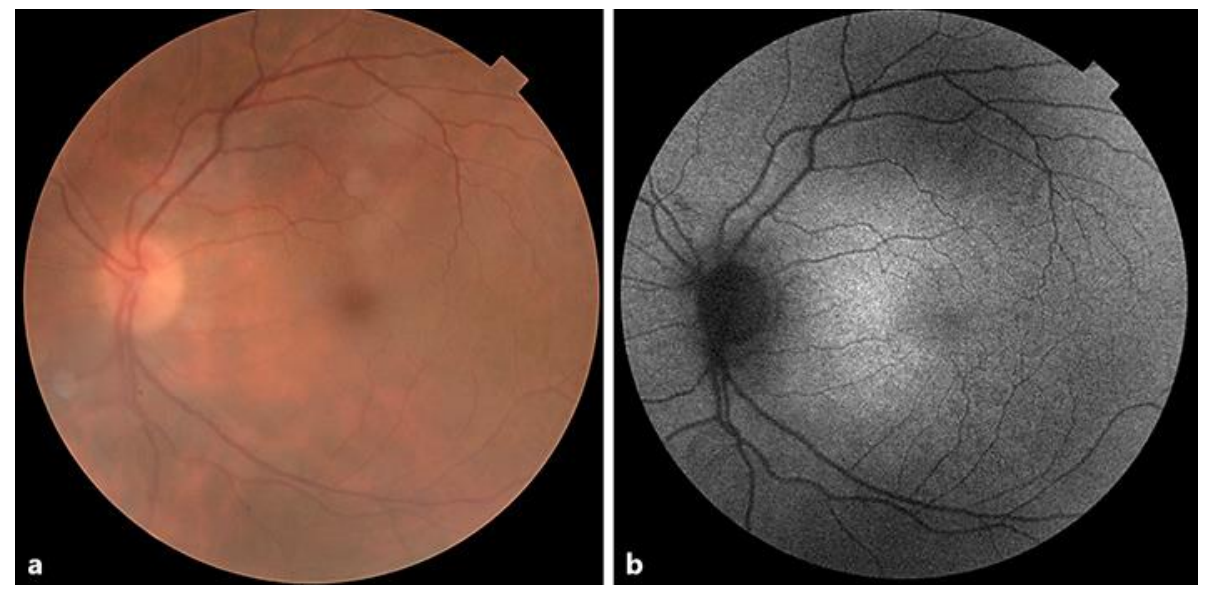

Fig. 3. Upon recurrence of inflammation, diffuse multiple small tiny creamish subretinal lesions were noted along with early disc swelling nasally (a). Fundus autofluorescence failed to detect any pathology (b). 


\section{Case Reports in Ophthalmology}
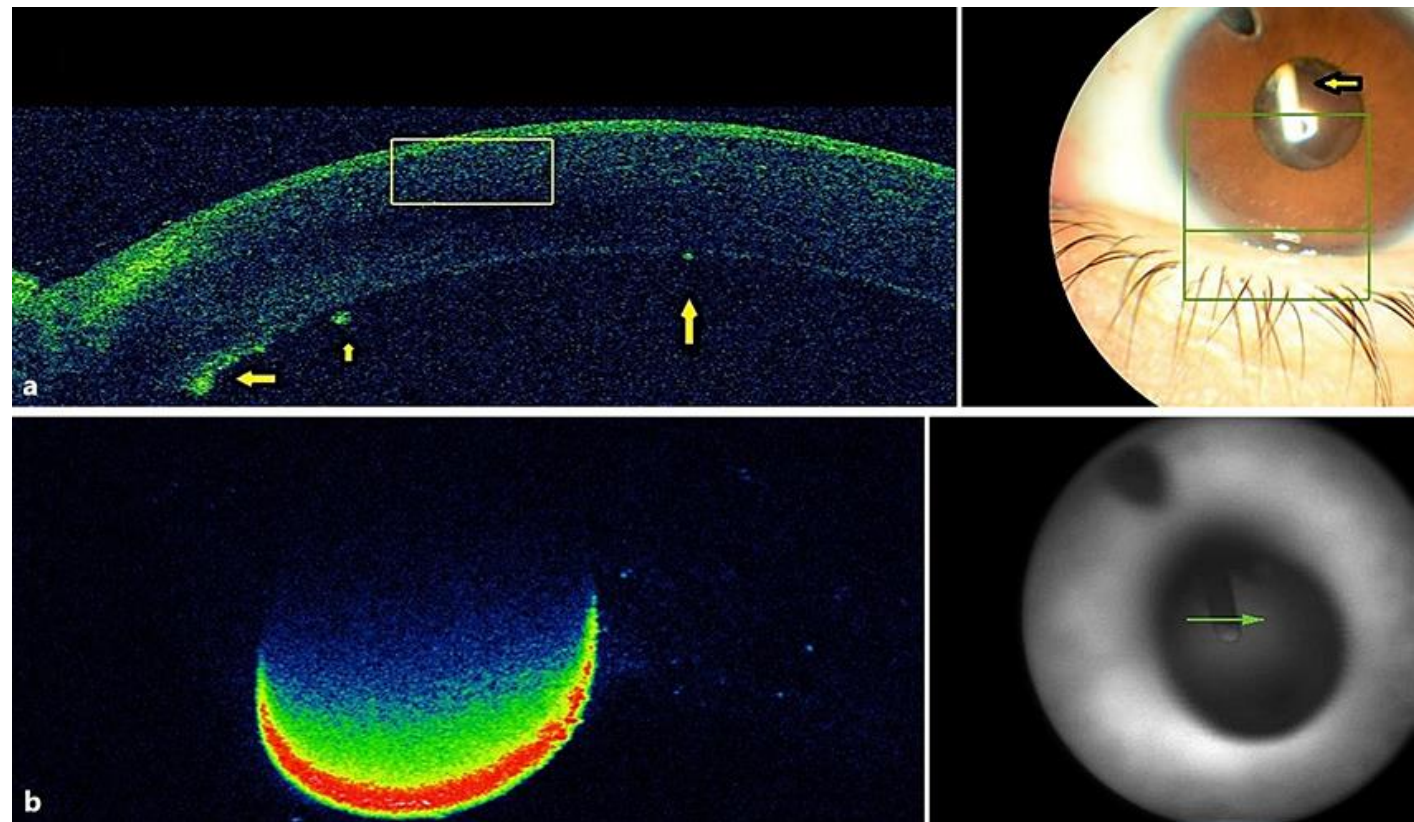

Fig. 4. Anterior OCT (Topcon 3D OCT-2000 FA plus, Topcon Inc., Tokyo, Japan) of the cornea (a) showed new onset of round keratic precipitates (arrows) as one of the clinical signs of recurrence of inflammation. Anterior OCT localized the position of the biodegradable dexamethasone implant (b). Note the superior peripheral iridectomy. OCT, optical coherence tomography. 